\title{
Facial Expression Recognition using Save and Load Keywords
}

\author{
Niranjan Bhattacharyya, $\mathrm{PhD}$ \\ HOD, Department of ECE, \\ BPIT, PSP-4, Sector -17, Rohini, \\ Delhi, India - 110089
}

\author{
Anshul Sharma \\ BPIT, affiliated to GGSIP University \\ 167, AgrohaKunj, Sector-13, Rohini, \\ Delhi, India - 110085
}

\begin{abstract}
Human expression is one of the most used gesture to express and understand the true intentions of a person. Recognition of human facial expression can be very useful in lie detection. It is mainly used for security reasons so that human behavior can be determined more efficiently, which is difficult for a human eye to detect. This paper focusses on the problem of facial expression recognition and suggests how to make it more efficient and faster. Facial expression code usually takes very long time to load all the database images to which current images can be matched. This problem will be solved by using 'Save' [1] and 'Load' [8] keywords in the Matlab code for expression recognition. Findings have shown that the code execution was around seventeen percent faster with the use of 'Save' and 'Load' keywords.
\end{abstract}

\section{General Terms}

Facial Expression Recognition, Algorithms

\section{Keywords}

.mat, Facial Expression Recognition, Load, Principal Component Analysis, Save.

\section{INTRODUCTION}

Communication among humans can be verbal and nonverbal. Verbal communication can only provide a person an objective information. Whereas, nonverbal communication can provide a subjective information. Expression, therefore can be a really good source for extraction of true intentions and meaning. That is why facial expression recognition is a popular security tool now days.

There are six basic human expressions [11] which are used in day to day life. These expressions are Anger, Happiness, Sadness, Disgust, Surprise and Fear. And these expressions can be recognized through code by analyzing the different positions of human eye, nose, eyebrows and mouth. To recognize expressions, the actual picture of the person is captured at the moment and it is matched with the images that we have stored in database prior to the tests. These database images comprises of all the six basic expressions from all around the world. As different cultures have different way of expressing certain emotions, database images need to cover a wide area.

Many new technologies and methods have now been introduced to recognize facial expressions more efficiently. Some of them are Principal Component Analysis (PCA) [5], Independent Component Analysis (ICA) [9], Double Principal Component Analysis and more.

These all methods still need to have database images so that expressions can be extracted by matching the photos to these database images. During the execution of Facial Expression Recognition code through Matlab, all of these database images have to be loaded into the software. As we need software to be efficient to recognize all the type of expression and we need to cover expressions of various cultures and for this we require a large number of database images. Loading a large number of database image in the program would cause delay in program execution, as all the database images will be loaded into the code one by one, every time we run the program. This would take a lot of time, as minimum 300 images have to be loaded in our code to efficiently recognize the expressions. Save and Load command can help out here to skip the part of loading images every time we start of program.

\section{FACIAL EXPRESSION IMAGE DATABASE}

Facial expression image database consists of preloaded images. These images are of four people in 6 basic expressions in a day light condition. The image dataset consists of some other suggested pictures too, which are of different people belonging from different cultures to have a wide variety in the image dataset. Now, each one of database images has not to be loaded every time to program is started. These all images can be converted in '.mat' format and loaded at once. This concept can be implemented irrespective of the technology used. This can be used with PCA [5], ICA [9] or Double PCA

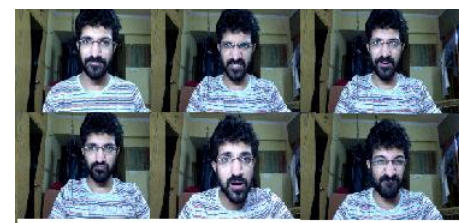

mat file

Fig 1: Multiple database images being converted to a single .mat file

\section{BASIC PRINCIPLE}

Each database image is necessary to compare the captured picture. So all the images must be loaded to make an efficient comparison. Loading a large number of images would take a lot of time to complete the program. The idea suggested here is that all the database should be converted to a single file. So that only that one file will be loaded whenever the program will run. This would improve the processing speed of the program and won't spend too much time on loading each database image. This can be done through 'Save' and 'Load' keywords, which are present in Matlab.

Suppose we have all the database images in a 'Database_Images' folder. We change the folder location to 'Database_Images' folder in Matlab and write the following command:

\section{Save Db_Images.mat}

As shown in Fig 1 also, this command would create a 'Db_Images.mat' file in the same location where all the database photos were present. '.mat' extension files are binary 
data container files which can store images, variables, arrays and other information which has to be used in Matlab.
After creating '.mat' file for all the database images we can replace the part of code where all the images were loaded with just one line.

Load Db_Images.mat

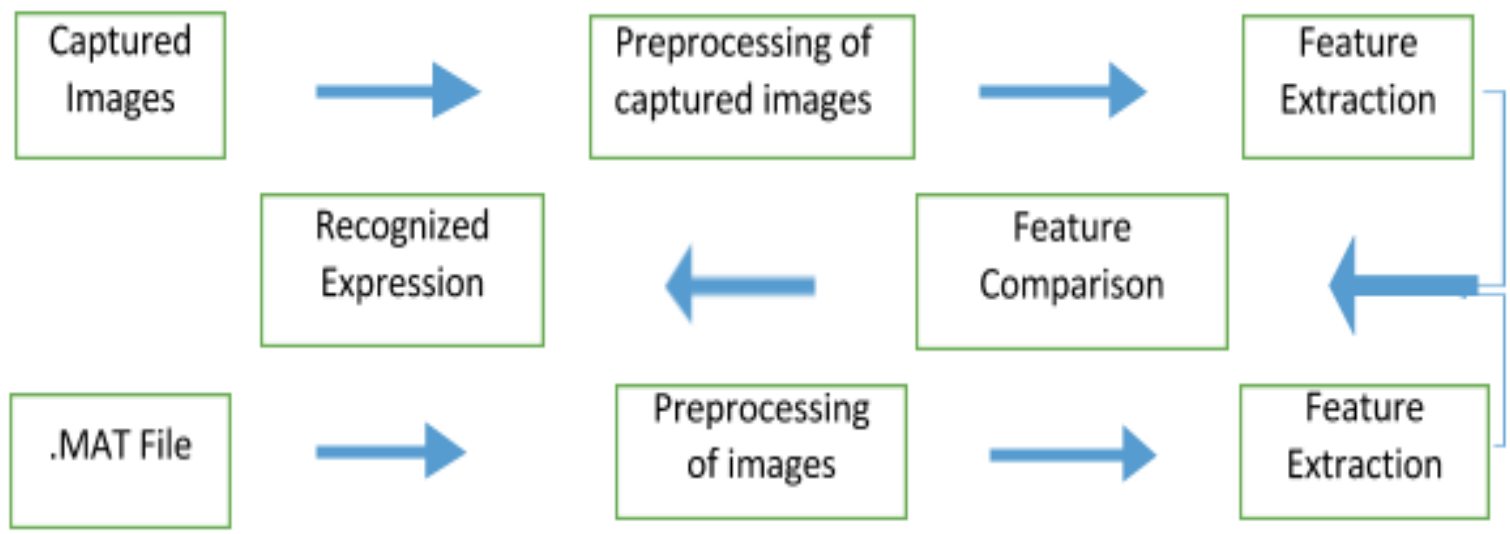

Fig 2: Workflow for the Facial Expression Recognition code

\section{EXPERIMENTAL RESULTS}

This 'load Db_Images.mat' would skip the loading of each database image and would load all the images in a considerable short time. This would eventually reduce the processing speed. This would also reduce the code length as the loading part of all images is now replaced with just one command.

The current images, whose expressions are to be recognized are 10 . And the database images through which expression is to be recognized are around 325 . The code is run 5 times and the time recorded with and without 'Save' and 'Load' commands for the program completion when all the conditions are set same is specified in table and the chart.

Table 1. Experimental results of time taken by the code to execute completely when executed with and without 'Save' and 'Load' keywords

\begin{tabular}{|c|c|c|}
\hline \multirow{2}{*}{ Tests } & \multicolumn{2}{|c|}{ Time Taken } \\
\cline { 2 - 3 } & $\begin{array}{c}\text { Without 'Save' and } \\
\text { 'Load' keyword }\end{array}$ & $\begin{array}{c}\text { With 'Save' and } \\
\text { 'Load' keyword }\end{array}$ \\
\hline Test 1 & $\begin{array}{c}5 \text { minutes and 5 } \\
\text { seconds }\end{array}$ & 52 seconds \\
\hline Test 2 & $\begin{array}{c}5 \text { minutes and } 8 \\
\text { seconds }\end{array}$ & 53 seconds \\
\hline Test 3 & $\begin{array}{c}5 \text { minutes and 3 } \\
\text { seconds }\end{array}$ & 52 seconds \\
\hline Test 4 & $\begin{array}{c}4 \text { minutes and 59 } \\
\text { seconds }\end{array}$ & 55 seconds \\
\hline Test 5 & $\begin{array}{c}5 \text { minutes and } 8 \\
\text { seconds }\end{array}$ & 54 seconds \\
\hline
\end{tabular}

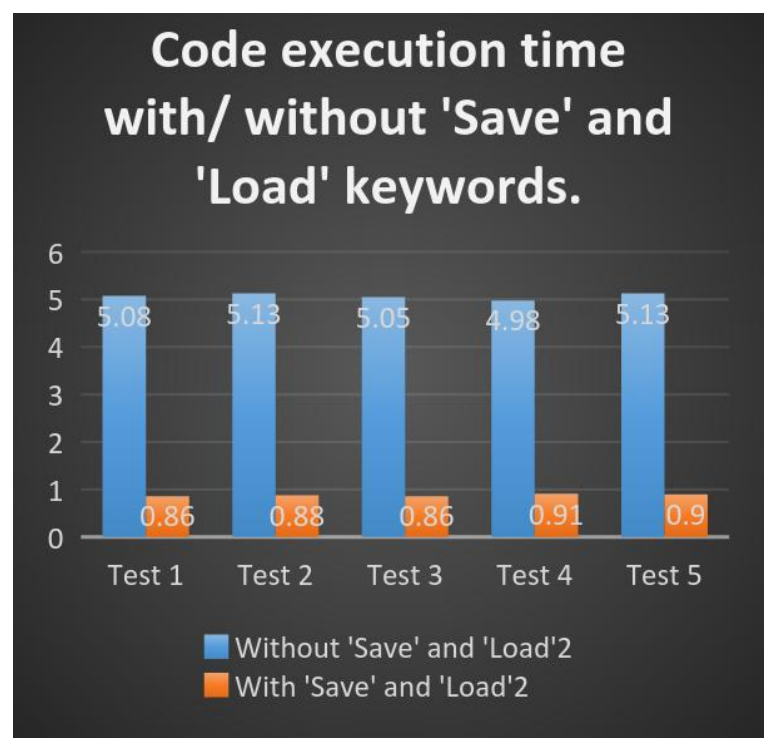

Fig 3: Graphical representation of code execution time in minutes vs. tests when executed with and without 'Save' and 'Load' keywords.

\section{CONCLUSION}

The new approach to recognize facial expression code using 'Save' and 'Load' keywords reduces the execution time of the code to a considerably good level. This also optimizes the code by reducing the code length. The time taken by the code to completely execute was dropped by around seventeen percent, when all the condition including database images and current captured images were kept same. This would increase the performance of any expression recognition software implemented using different methods. The performance of code can be further improved by increasing the size of database images and converting it into a .mat file. This would also help in recognizing a particular expression. 


\section{REFERENCES}

[1] Save keyword description by Mathworks, http://in.mathworks.com/help/matlab/ref/save.html.

[2] Journal on Facial Expressions Analysis by Ying-Li Tian, Takeo Kanade, and Jeffrey F. Cohn.

[3] M. Turk and A. Pentlnd, "Eigenfaces for Recognition," J. CognotiveNueroscience, vol.3, no.1 pp. 71-86, 1991.

[4] Lindsay I Smith. "A Tutorial on Principal Component Analysis".

[5] Facial Expression Recognition using PCA by DebasmitaChakrabarti and Debtanu Dutta.

[6] Save, Load and Delete Workspace Variable by Mathworks, http://in.mathworks.com/help/matlab/matlab_env/save-loadand-delete-workspace-variables.html.
[7] Difference between .m and .mat file by Andreas Goser, https://in.mathworks.com/matlabcentral/answers/27048difference-between-m-and-mat-files.

[8] Load keyword description by Mathworks, http://in.mathworks.com/help/matlab/ref/load.html.

[9] Recognizing Facial Expressions with PCA and ICA onto dimension of the emotion by Young-suk Shin.

[10] Recognizing faces with PCA and ICA by Bruce A.Draper, KyungimBaek, Marian Stewart Barlett and J. Ross Beveridge.

[11] The Universally Recognized Facial Expressions of Emotions by Cole Calistra. 